\title{
A New Method to Evaluate Residual Flux Thanks to Leakage Flux, Application to a Transformer
}

\author{
Didier Cavallera $^{1}$, Vinicius Oiring ${ }^{2}$, Jean-Louis Coulomb ${ }^{1}$, Olivier Chadebec ${ }^{1}$, \\ Bruno Caillault ${ }^{2}$, and Francois Zgainski ${ }^{2}$ \\ ${ }^{1}$ Grenoble Electrical Engineering Laboratory, University of Grenoble, UJF, CNRS, Grenoble 38185, France \\ ${ }^{2}$ EDF/DTG, EDF Group-Hydro Generation and Engineering, Grenoble 38185, France
}

\begin{abstract}
This paper presents a new method to estimate the residual flux in the magnetic circuit of a transformer, which is the key data to determine the ideal closing time for the re-energization of a transformer. The methodology described is based on the measurement with fluxgate sensors of the magnetic field induced by the leakage flux around a single-phase transformer.
\end{abstract}

Index Terms-Magnetic field measurement, magnetic flux leakage, remanence, transformers.

\section{INTRODUCTION}

D URING the re-energization of unloaded power transformers, undesirable effects (inrush currents, overvoltages, dynamical stress, resonances, etc.) can appear and may cause many problems in power system, mainly in transformers [1]. The determination of residual flux allows calculating the best closing time for the re-energization of a power transformer. There are many manufacturers who develop controlled switching. Transformer controlled switching is used for reducing the magnitude of the inrush currents [2], [3]. There are several ways to calculate the residual flux.

Nowadays, the mostly used method is voltage integration during the de-energization of power transformers; however, the determination of the residual flux can suffer from inaccuracies. Indeed, the measurement of the residual flux is uncertain due to low-voltage signal including dc component and noise. Furthermore, the influence on the residual flux of system transients near transformer flux has been recently underlined [4].

Another method consists in using the maximal current value; the principle is to calculate the residual flux from inrush current obtained when the transformer is re-energized [5]. This method is inappropriate for industrial applications, because the value of the residual flux is known only after the re-energization of the transformer. On the other hand, it is an excellent method to check the value of the residual flux.

This paper proposes a new method to calculate the residual flux from near magnetic field measurements, just before the energization of the transformer. The main advantage of this new method is to consider all possible changes of the residual flux (between the de- and re-energization of the transformer), because the residual flux is obtained in real time just before the energization of the transformer. Section II presents the link between the leakage flux measured near a transformer and the

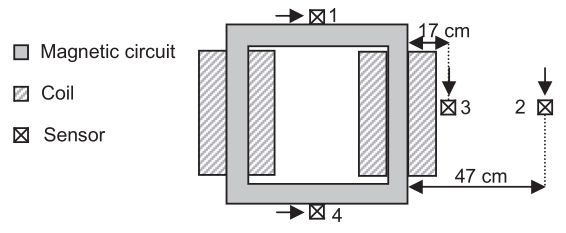

Fig. 1. Schematic view of the device.

residual flux in the magnetic circuit. Section III describes the new method for estimating the residual flux.

\section{LinK BetweEn THE LEAKAgE FLuX MEASURED NEAR A TRANSFORMER AND THE RESIDUAL FLUX OF A MAGNETIC CIRCUIT}

A single-phase transformer $(400 / 4 \mathrm{~V}, 32 \mathrm{kVA}$, two limbs) is chosen for this paper. Some of the magnetic sensors are located outside the magnetic circuit of the transformer (without tank in these tests). In Fig. 1, a schematic view of the transformer and some of the sensors positions are exposed.

The fluxgate technology sensor is chosen for its accuracy and static and dynamic measure capability. The measurement range of the sensor (fluxgate) is $\pm 100 \mu \mathrm{T}$ and the bandwidth is dc up to $3 \mathrm{kHz}$. For each sensor, only the component of the magnetic field tangential to the magnetic circuit is considered.

\section{A. Leakage Flux Measured Near a Transformer}

The test system consists of re-energizing this unloaded transformer. In [6], we realized some finite-element calculations that proved the existence of a leakage flux around the transformer when it is off line. The leakage flux is measured by sensors. Some energizations and de-energizations of the transformer with a voltage level of $400 \mathrm{~V}_{\mathrm{RMS}}$ are realized [Fig. 2(a)]. After the energization shown in circle 2 [Fig. 2(b)], we can observe that the average dc-component induction has changed on both opposite sensors (1 and 4), compared with the state before the energization (circle 1). It shows that the magnetic field in the transformer magnetic circuit has changed and creates a various leakage flux outside. The goal of this paper is to propose a method based on this magnetic field 

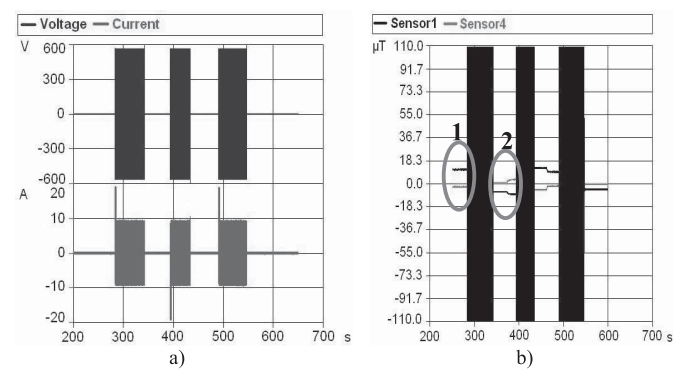

Fig. 2. Voltage, current, and induction measured during some different switching operations. (a) Voltage and current. (b) Sensors 1 and 4.
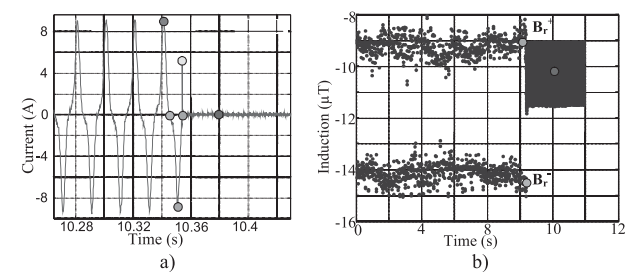

Fig. 3. Current measured and induction interpolated during test. (a) Current. (b) B Bensors 2 at zero current.

measure. Before, we must check the possibility to extract residual flux from the leakage flux.

\section{B. Distinction of Residual Flux Because of the Leakage Flux}

When the transformer is unloaded at nominal voltage (400 $\mathrm{V}_{\mathrm{RMS}}$ ), the measured field by one sensor is the sum of the magnetic fields induced from magnetic circuit leakage, the coil field, and the earth's magnetic field. The main issue is to extract the field created by the magnetic circuit leakage from the total field. To extract this field, an algorithm has been developed. For zero current values, the field induced by the coil is equal to zero; at these times, the total induction measured by a sensor is interpolated. An example of this method is shown in Figs. 3 and 4.

The curve $B(I)$, shown in Fig. 4, has not a conventional rotation sense, because it is not the induction measured inside the magnetic material but an induction measured in the air. This phenomenon is explained in Section II-C. In Fig. 4, two steps corresponding, respectively, to the points: $\mathrm{B}_{\mathrm{r}}^{+}$[point (4) or green] and $\mathrm{B}_{\mathrm{r}}^{-}$[point (2) or orange] [Fig. 3(b)]. When the de-energization occurs, the current goes to a maximum negative value [point (3) or blue on the Fig. 3(a)] and then realizes a short incursion in the positive part [point (5) or yellow]. Then, the residual flux takes a value between $\mathrm{B}_{\mathrm{r}}^{+}$and $\mathrm{B}_{\mathrm{r}}^{-}$[point (6) or red] following what is classically called a reverse curve. The current measurement is noisy, by consequence, the determination of the induction at zero current is difficult [especially the determination of the red point on Fig. 3(b)]. The final value of the induction is $-10 \mu \mathrm{T}$ (mean value after the de-energization). The ambient induction value measured is near $-12 \mu \mathrm{T}$ (mean value between the green and orange points). The residual flux creates on this sensor a field of $+2 \mu \mathrm{T}$, this result is

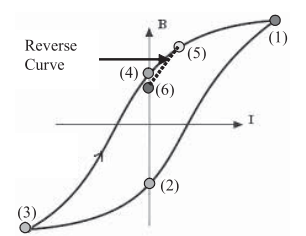

Fig. 4. Schematic view of a curve $B(I)$.

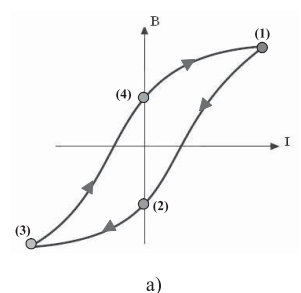

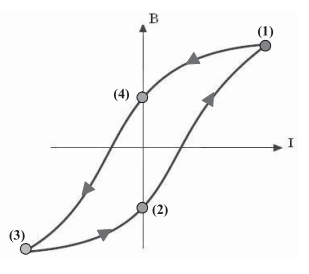

b)
Fig. 5. Schematic view of curves $B(I)$. (a) Induction in the air (sensor 2). (b) Induction in the magnetic circuit.

obtained by the difference between the final value of the induction $(-10 \mu \mathrm{T})$ and the ambient induction value $(-12 \mu \mathrm{T})$. We succeed in characterising the different field effects and we isolate the residual flux effect because of one field measurement.

\section{Electromagnetic Modeling of the Offline Transformer}

The main objective of the modeling consists in explaining the unconventional rotation sense of the curve $B(I)$ in Fig. 4 or Fig. 5(a).

When the current reaches its maximum level (1), the induction measured in the air (experimental measurement with sensor) or the magnetic circuit (theory) is positive and maximum. The next step is different for both curves. When the current is equal to zero, the induction is positive in Fig. 5(b) [point(4)] and negative in Fig. 5(a) [point (2)]. To sum up, when the current is zero, the sign of the induction in the magnetic circuit is opposite to the sign of the induction in the air. Because of a simple model to explain the leakage flux measured around an offline transformer [6], we can clarify this phenomenon.

The magnetic circuit has been modeled by some magnets, each one of them having a particular direction of magnetization [Fig. 6(a)]. An imposed residual induction is set to $1 \mathrm{~T}$ and the material relative permeability is 5000. To consider the magnetic circuit air gaps, in the corners of the structure, four air gaps are placed with a thickness of $0.5 \mathrm{~mm}$.

FEM simulations enable to see that for this configuration, the field created out of the transformer is not equal to zero. In particular, we can see [Fig. 6(b)] that the sign of the induction in the magnetic circuit (circle A) is opposite to the induction in the air where sensor 2 is placed (circle $\mathrm{B}$ ).

Therefore, the curve $B(I)$ presented in the $\S I I-\mathrm{B}$ has not a conventional sense. 


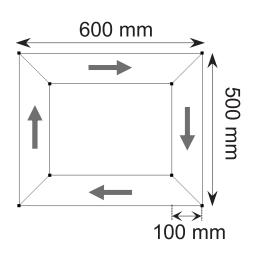

a)

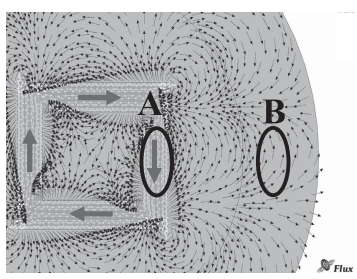

b)
Fig. 6. Magnetic circuit modeling with air gaps in the corner. (a) Transformer modeling. (b) FEM simulation results.
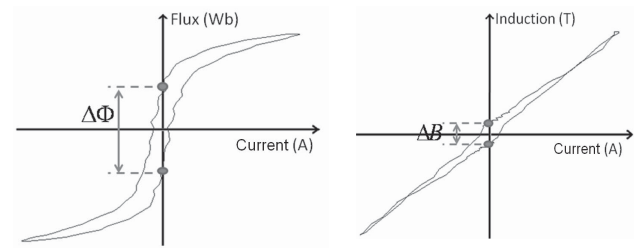

Fig. 7. Parameters of transfer function $(\Delta \Phi, \Delta \mathrm{B})$.

\section{Methods to Evaluate Residual FluX IN A TRANSFORMER}

\section{A. New Method to Estimate the Residual Flux}

The method proposed consists in estimating the residual flux from a magnetic field measurement sensor (fluxgate), installed permanently at a fixed location, just before the energization. To do this, we propose a two-step approach.

The first step is to establish the transfer function between induction and flux. This first step can be achieved when the transformer is supplied at its nominal voltage. The parameters of the transfer function are shown in Fig. 7.

The induction is measured by a magnetic field sensor and the flux is obtained from the integration of the voltage, measured directly on the secondary winding of the transformer. From these two signals (flux and induction), it is possible to determine the transfer function using one or more periods (20 ms) for each signal. In other words, for a period, we obtain one pair of values for each signal (flux and induction) when the current is equal to zero. By doing the subtraction of each pair of values (flux and induction), we can obtain delta flux $(\Delta \Phi)$ and delta induction $(\Delta \mathrm{B})$, the division between $(\Delta \Phi)$ and $(\Delta \mathrm{B})$ is the transfer function.

As the locations of the sensors are fixed, the determination of the transfer function must be done only one time.

The second step is to measure the induction when the transformer is offline, just before each re-energization. With this signal, we can obtain the value of the residual induction $\left(\Delta \mathrm{B}_{\mathrm{r}}\right)$; see Fig. 8 .

To do this, it is necessary to do the subtraction between the mean values of the induction, measured when the transformer is offline and online, respectively. This value $\left(\Delta \mathrm{B}_{\mathrm{r}}\right)$ corresponds to the residual flux in the transformer. Finally, to get the residual flux $\left(\Delta \Phi_{\mathrm{r}}\right)$, we simply multiply the residual induction by the transfer function. This equation is shown hereafter

$$
\Delta \varphi_{r}=\Delta B_{r} \times \frac{\Delta \varphi}{\Delta B} .
$$

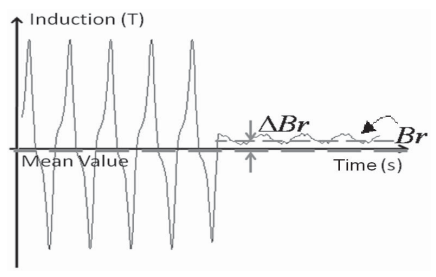

Fig. 8. Delta residual induction $(\Delta \mathrm{Br})$.

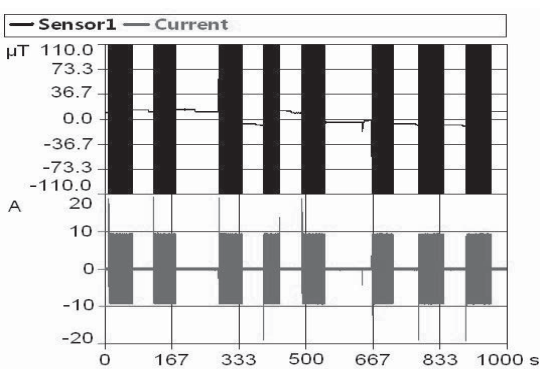

Fig. 9. Magnetic field and current measured for a sequence of eight energizations.

TABLE I

Residual Flux Calculated by Different Methods

\begin{tabular}{|c|c|c|c|}
\hline $\begin{array}{c}\text { Before } \\
\text { Energization }\end{array}$ & $\begin{array}{c}\text { Maximal } \\
\text { Current } \\
\text { Method (\%) }\end{array}$ & $\begin{array}{c}\text { Voltage } \\
\text { Integration } \\
\text { Method (\%) }\end{array}$ & $\begin{array}{c}\text { New } \\
\text { Method } \\
\text { sensor 1 (\%) }\end{array}$ \\
\hline 1 & -18 & -18 & -18 \\
\hline 2 & -18 & -15 & -13 \\
\hline 3 & 17 & 19 & 26 \\
\hline 4 & -18 & -18 & -17 \\
\hline 5 & 8 & 15 & 8 \\
\hline 6 & 17 & 18 & 18 \\
\hline 7 & 17 & 20 & 16 \\
\hline 8 & -18 & -16 & -27 \\
\hline
\end{tabular}

\section{B. Application With a Sensor Located on the Magnetic Circuit}

In this section, we present the results obtained for the singlephase transformer presented. It is necessary to remember that the magnetic field sensor (sensor 1) is located on the magnetic circuit. Its goal is to obtain an accurate residual induction measurement. A key point for this configuration is the accuracy of current measurement when the current is equal to zero. The signals of induction and current are shown in Fig. 9 for a sequence of eight cycles of energizations (the instants of energization correspond to the maximum voltage).

In Fig. 9, we can observe the relation between residual induction measurement, $\Delta \mathrm{B}_{\mathrm{r}}$, when the transformer is offline and the signal of the inrush current. The behavior of these parameters can be explained by

$$
\text { if }\left\{\begin{array}{l}
\Delta B r_{n} \geq 0 \rightarrow I_{n+1} \leq 0 \\
\Delta B r_{n} \leq 0 \rightarrow I_{n+1} \geq 0
\end{array}\right.
$$

where $\mathrm{Br}$ is the residual flux in the transformer, $I$ is the inrush current, and $n$ is the number of the energization (1-8).

Table I presents the results for the residual fluxes calculated for the sequence of eight energizations shown in the Fig. 9. The results are expressed in percentage of the nominal flux of the transformer $(1.8 \mathrm{~Wb})$.

The method of determination of the transfer function has been applied for each operation, to test its robustness. NB: as 


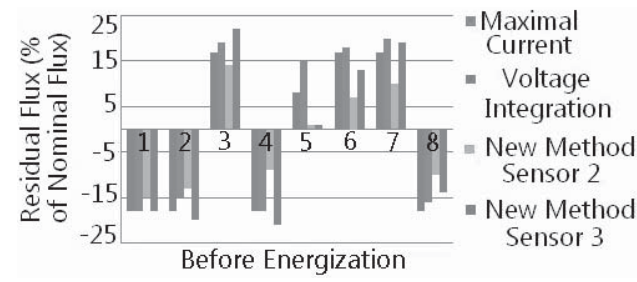

Fig. 10. Residual flux calculated by different methods.

it has been previously explained, the transfer function should be determined once, for the magnetic field sensor used.

The new method is consistent with the maximal current method [5] in five of the eight cases of energization. The new method presents wrong results regarding the integration voltage method [3] for cases 2, 3, and 8. The noise in the current signal leads to uncertainties in the determination of the transfer function. On the other hand, the new method is more efficient than voltage integration method for case 5. In this case, there has been an operation resulting in a modification of the residual flux in the transformer after its switching off. This resulting evolution of the flux cannot be detected by the voltage integration method, unless calculating the integration during all the period in which the transformer is out of power, which would be very inaccurate.

The new method considers all possible changes of the residual flux, because the delta residual induction $\left(\Delta \mathrm{B}_{\mathrm{r}}\right)$ considered is obtained in real time just before the energization of the transformer. This is one of the main advantages of our method, despite its relative inaccuracy in some of the cases. Further improvements in current measurement and data processing are being undergone.

\section{Application for Sensors Located Outside of the Transformer}

Knowing that the environment inside a real transformer is strongly aggressive for a magnetic field sensor in terms of corrosion (transformer oil) and temperature, we are still interested in developing a noninvasive method (sensor located outside of the transformer), as in [7].

A key point for this configuration is the position of the magnetic field sensors, because the magnitude of the induction signal decreases considerably in function of the distance. Another important characteristic of this configuration is that the induction signal will be sensitive to any kind of possible noises (environmental magnetic field).

In this section, we will present the results obtained for the magnetic field sensors outside of the transformer. It is necessary to remember that the magnetic field sensors (sensors 2 and 3) are placed at 47 and $17 \mathrm{~cm}$ from the singlephase transformer without tank, respectively (Fig. 1). Therefore, this section will present important limitations regarding to a real transformer.

Fig. 10 shows the results for the residual fluxes calculated for the sequence of eight energizations shown in Fig. 9.

On the one hand, the new method for sensor 2 presents inaccurate results regarding to the integration voltage and the maximal current methods for all the cases. The main reason for this is that sensor 2 is farther than sensor 3 from the magnetic circuit. For this sensor, the main part of induction signal measurement is noised (environmental magnetic field) and the minor part is the residual induction, which corresponds to the residual flux present in the magnetic circuit. Therefore, the results obtained for sensor 2 will present less accuracy.

On the other hand, for sensor 3, the new method presents better results regarding the maximal current and voltage integration methods. Indeed, for sensor 3, the main part of the induction signal measurement is the residual induction.

Therefore, for sensors located outside of the transformer, it is necessary to pay attention to the distance between the sensor and the magnetic circuit.

\section{CONCLUSION}

In this paper, a new method to estimate the residual flux in the magnetic circuit of a transformer is presented. Magnetic field measurements are done with magnetic field sensors near and far from the transformer. We calculate the residual flux value by isolating the magnetic field induced by the leakage flux. The new method of residual flux computation gives consistent results for a sensor on the magnetic circuit and coherent results for a sensor outside the magnetic circuit. The key points for the new method are the current measurement quality for small values (near zero) and the distance between the sensor and the magnetic circuit. As the measurements are done just before each re-energization, the major advantage of this new method regarding other methods (integration voltage and maximal current) is that it considers all possible changes of the residual flux in the magnetic circuit. To sum up, this method is straightforward and presents a rather good agreement with the existing methods. In future work, we will focus on its application to three-phase transformers, considering different core topologies.

\section{REFERENCES}

[1] M. Steurer and K. J. Fröhlich, "The impact of inrush currents on the mechanical stress of high voltage power transformer coils," IEEE Trans. Power Del., vol. 17, no. 1, pp. 155-160, Jan. 2002.

[2] Y. Cui, S. G. Abdusalam, S. Chen, and W. Xu, "A sequential phase energization technique for transformer inrush current reduction-Part I: Simulation and experimental results," IEEE Trans. Power Del., vol. 20, no. 2, pp. 943-949, Apr. 2005.

[3] J. H. Brunke and K. J. Fröhlich, "Elimination of transformer inrush currents by controlled switching. I. Theoretical considerations," IEEE Trans. Power Del., vol. 16, no. 2, pp. 276-280, Apr. 2001.

[4] Y. Corrodi, K. Kamei, H. Koyama, H. Ito, and T. Goda, "Influence of system transients on the residual flux of an unloaded transformer," in Proc. IEEE Power Energy Soc. General Meeting, Jul. 2011, pp. 1-7.

[5] R. Yacamini and A. Abu-Nasser, "Numerical calculation of inrush current in single phase transformers," IEE Proc. B, Electr. Power Appl., vol. 128, no. 6, pp. 327-334, Nov. 1998.

[6] D. Cavallera, J.-L. Coulomb, O. Chadebec, B. Caillault, and F.-X. Zgainski, "A simple model to explain the leakage flux measured around an off line transformer," in Proc. 18th Int. Conf. Comput. Electromagn. Fields, Sydney, Australia, 2011.

[7] V. P. Bui, O. Chadebec, L.-L. Rouve, and J.-L. Coulomb, "Noninvasive fault monitoring of electrical machines by solving the steadystate magnetic inverse problem," IEEE Trans. Magn., vol. 44, no. 6 , pp. 1050-1053, Jun. 2008. 